\title{
Monitoring Plastic-Mulched Farmland by Landsat-8 OLI Imagery Using Spectral and Textural Features
}

\author{
Hasituya ${ }^{1}$, Zhongxin Chen ${ }^{1, *}$, Limin Wang ${ }^{1}$, Wenbin $\mathrm{Wu}^{1}$, Zhiwei Jiang ${ }^{2}$ and $\mathrm{He} \mathrm{Li}^{1}$ \\ 1 Key Laboratory of Agri-informatics, Ministry of Agriculture/Institute of Agricultural Resources and \\ Regional Planning, Chinese Academy of Agricultural Sciences, No. 12, Zhongguancun Nan Dajie, \\ Beijing 100081, China; 2009hasituya@sina.com (H.); wanglimin01@caas.cn (L.W.); \\ wuwenbin@caas.cn (W.W.); lihe_caas@163.com (H.L.) \\ 2 National Meteorological Information Center, China Meteorological Administration, No. 46, \\ Zhongguancun Nan Dajie, Beijing 100081, China; zhiweij@@163.com \\ * Correspondence: chenzhongxin@caas.cn; Tel.: +86-10-8210-5089
}

Academic Editors: Ioannis Gitas and Prasad S. Thenkabail

Received: 4 February 2016; Accepted: 14 April 2016; Published: 22 April 2016

\begin{abstract}
In recent decades, plastic-mulched farmland has expanded rapidly in China as well as in the rest of the world because it results in marked increases of crop production. However, plastic-mulched farmland significantly influences the environment and has so far been inadequately investigated. Accurately monitoring and mapping plastic-mulched farmland is crucial for agricultural production, environmental protection, resource management, and so on. Monitoring plastic-mulched farmland using moderate-resolution remote sensing data is technically challenging because of spatial mixing and spectral confusion with other ground objects. This paper proposed a new scheme that combines spectral and textural features for monitoring the plastic-mulched farmland and evaluates the performance of a Support Vector Machine (SVM) classifier with different kernel functions using Landsat- 8 Operational Land Imager (OLI) imagery. The textural features were extracted from multi-bands OLI data using a Grey Level Co-occurrence Matrix (GLCM) algorithm. Then, six combined feature sets were developed for classification. The results indicated that Landsat-8 OLI data are well suitable for monitoring plastic-mulched farmland; the SVM classifier with a linear kernel function is superior both to other kernel functions and to two other widely used supervised classifiers: Maximum Likelihood Classifier (MLC) and Minimum Distance Classifier (MDC). For the SVM classifier with a linear kernel function, the highest overall accuracy was derived from combined spectral and textural features in the $90^{\circ}$ direction $(94.14 \%$, kappa 0.92$)$, followed by the combined spectral and textural features in the $45^{\circ}(93.84 \%$, kappa 0.92$), 135^{\circ}(93.73 \%$, kappa 0.92$), 0^{\circ}(93.71 \%$, kappa 0.92) directions, and the spectral features alone (93.57\%, kappa 0.91). Spectral features make a more significant contribution to monitoring the plastic-mulched farmland; adding textural features from medium resolution imagery provide only limited improvement in accuracy.
\end{abstract}

Keywords: plastic-mulched farmland; spectral features; textural features; support vector machine; Landsat-8; OLI imagery

\section{Introduction}

The recent agricultural practice of using plastic coverings is one of the most important differences between traditional agriculture and intensive agriculture. Plastic mulching is a technique used on a wide variety of crops, fruits and vegetables to provide protection from unfavorable growing conditions. In recent decades, the farmland covered by plastic film has increased considerably because such mulching has multiple functions in agricultural production: improving hydrothermal conditions, promoting crop growth, increasing crop yields, and mitigating the effects of drought and 
flooding, cold and heat, insects and diseases [1,2]. Plastic film has been used in many forms, such as greenhouses, medium and low tunnels and soil mulching. In China, the area of plastic-mulched farmland accounts for over $90 \%$ of total plastic-covered farmland. China has the largest area of plastic-mulched farmland in the world and that area has been growing rapidly; the 0.12 million ha covered by plastic film in 1981 rocketed to 19.79 million ha in 2011 [3] and increased to 25 million ha in 2013 [4]. However, the extensive use of plastic film in agriculture has been accompanied by a series of negative impacts on the climate, the eco-environment and the soil micro-environment. For example, plastic mulching changes the energy balance, water balance and carbon balance between the land surfaces, the atmosphere and the soil. Moreover, as plastic residues accumulate over years of use, the result is soil structure deterioration, farmland quality degradation, crop yield reduction, blocking of farming operations, and environmental pollution. Recently, such problems have become increasingly serious [3,5]. Nevertheless, because of the high dynamics of agronomic practices, there is no clear and accurate information concerning area coverage, spatial distribution or the temporal variation characteristics of plastic-mulched farmland in China. Various organizations and applications require timely information and a comprehensive understanding of the coverage and condition of plastic-mulched farmland. These include industries that plan and produce plastic film, organizations that recycle and provide oversight of plastic residues, and researchers studying land surface temperatures, evapotranspiration, crop phenology, and so on. Therefore, it is important to determine the spatiotemporal pattern and the magnitude of plastic-mulched farmland. Policy-makers and scientists urgently need effective methods to monitor and map the spatiotemporal pattern and distribution area of plastic-mulched farmland. Although conventional in-situ field investigation may provide accurate information about plastic-mulched farmland within small regions, field investigation is too labor-intensive, time-consuming and expensive for large regions. Moreover, annual report data tend to be subjective and lack space and time information; these mentioned methods are not sufficiently objective and do not result in timely and accurate reports.

Remote sensing is an effective technique for obtaining up-to-date information quickly and remotely over wide areas [6,7]. Using remote sensing, it is possible to obtain qualitative and quantitative information about ground objects [8]. However, relatively few research efforts so far have been conducted with a goal of monitoring and mapping plastic-mulched farmland using remote sensing. Wang et al. [9] discussed how to distinguish plastic-mulched paddy field under the water background using multi-angle polarization information. Lu et al. [10] extracted the plastic-mulched cotton farmland from Landsat-5 TM data using a decision tree classifier. Subsequently, they [11] developed a threshold model for monitoring transparent plastic-mulched cotton fields using MODIS (Moderate-resolution Imaging Spectroradiometer) time series data. All these methods provided ideal results. There were also some studies concerning detection of greenhouses and plastic-covered vineyards. Carvajal et al. [12], Agüera et al. [13], Agüera and Liu et al. [14], and Dilek et al. [15] extracted greenhouse information from very high spatial resolution imagery and Zhao et al. [16] from TM data using conventional supervised classification algorithms or machine learning algorithms, and all got ideal results.

From the literature review, we found that: (1) current studies for monitoring plasticulture focused largely on greenhouses, rarely on plastic-mulched farmland; and (2) most of the used data were high spatial resolution imagery, and the Landsat- 8 data have not been used. Only three papers discussed monitoring plastic-mulched farmland with remote sensing techniques and in these, only spectral information was used; other features such as textural or spatial features have not been discussed. It is currently unclear what types of remotely sensed data, what features, or what methods are most effective for monitoring plastic-mulched farmland.

Plastic mulching changes the surface textural characteristics in fact; thus, supplementing spectral features with textural features can theoretically enhance the separability of plastic-mulched farmland. However, there was no research to explain whether textural information can provide useful information for monitoring plastic-mulched farmland. Therefore, the overarching aim of this work was to find the optimal data source, features and methods. 
The first objective of this study was to examine whether Landsat-8 OLI data are feasible for monitoring plastic-mulched farmland.

The second objective was to determine the contribution of textural features for monitoring plastic-mulched farmland.

The third objective was to investigate the performance of SVM with different kernel functions and the performance of a non-parametric classifier by comparing them with maximum likelihood and minimum distance classifiers.

\section{Study Area and Data}

\subsection{Study Area}

This study was carried out in Jizhou, Hebei Province, China, which is an agricultural region located from $37^{\circ} 18^{\prime} 40^{\prime \prime} \mathrm{N}$ to $37^{\circ} 44^{\prime} 25^{\prime \prime} \mathrm{N}$ and from $115^{\circ} 09^{\prime} 57^{\prime \prime} \mathrm{E}$ to $115^{\circ} 41^{\prime} 07^{\prime \prime} \mathrm{E}$ (Figure 1) in the North China Plain. The study region has a warm temperate continental monsoon climate, with a dry and windy spring, hot and rainy summer, and a cold and dry winter. The area includes many types of soil, but sandy loamy and light loamy soil accounts for approximately $85.3 \%$ of all soil types. Because this area has sufficient rain and heat that appear in synchrony with the season, it is an appropriate region for growing and developing grain and cotton. The natural elements and extensive anthropogenic influences make it possible for land cover/use types within this area to be broadly classified into cultivated land, woodland, grassland, water body, traffic land, residential land, industrial land, etc. Jizhou occupies a $9.22 \times 10^{4}$ ha area. The terrain is mostly flat and tilts slightly from southwest to northeast. The average elevation ranges from $22 \mathrm{~m}$ to $27 \mathrm{~m}$. Farmland occupies $5.93 \times 10^{4}$ ha [17] (approximately $64.36 \%$ of the total area). The types of farmland include paddy fields, dry fields, vegetable fields, etc. The major crops in this area are cotton, winter wheat, corn and chili peppers. Cotton fields are mulched by white plastic film, and these plastic-mulched cotton fields form obvious spatial patterns in the field and in the imagery (Figure 2).

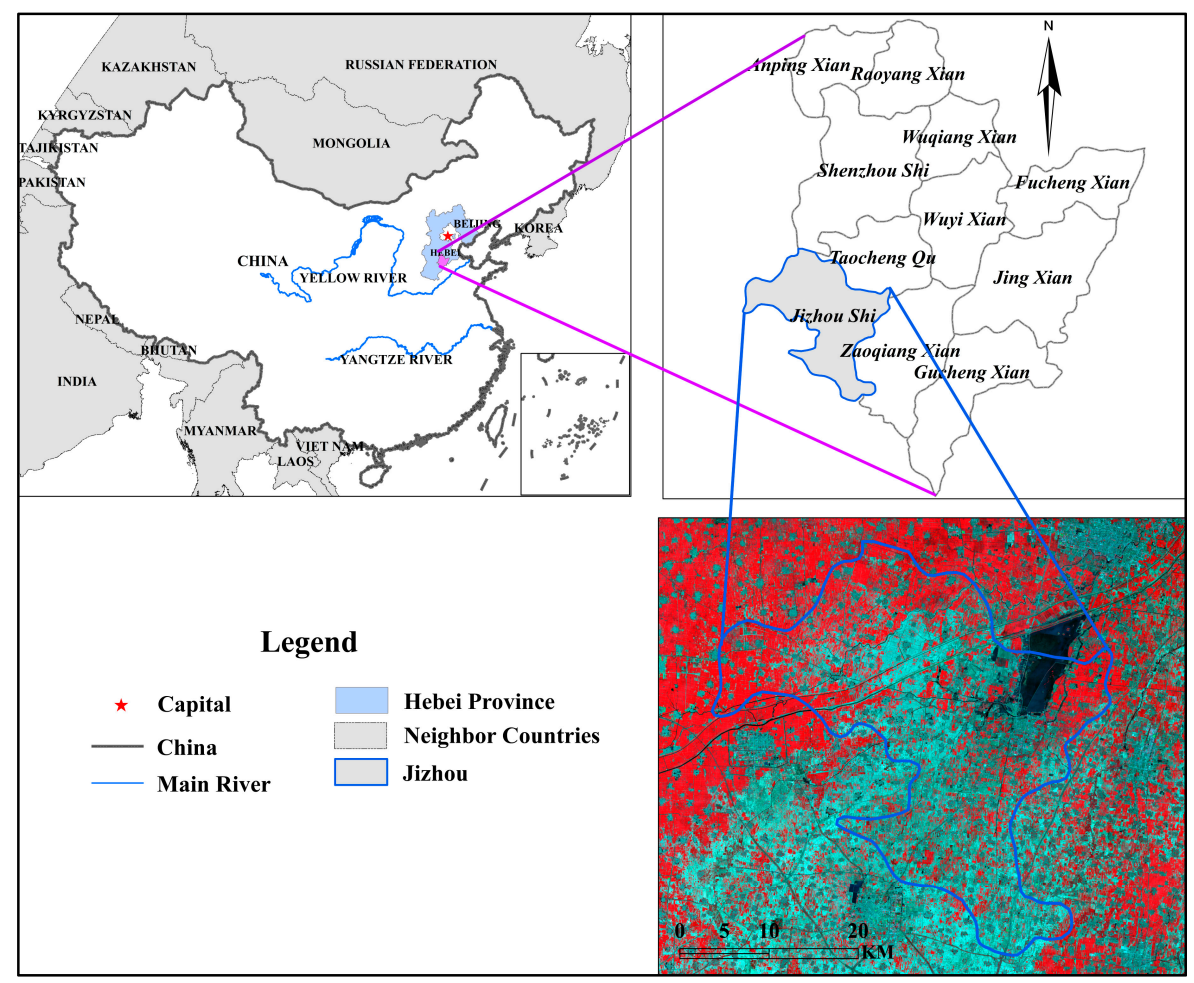

Figure 1. Location of the study area. (The imagery displayed in the figure is the Landsat OLI imagery presented in false color composite: $\mathrm{R}=$ NIR (Near Infrared), $\mathrm{G}=$ red, $\mathrm{B}=$ green). 

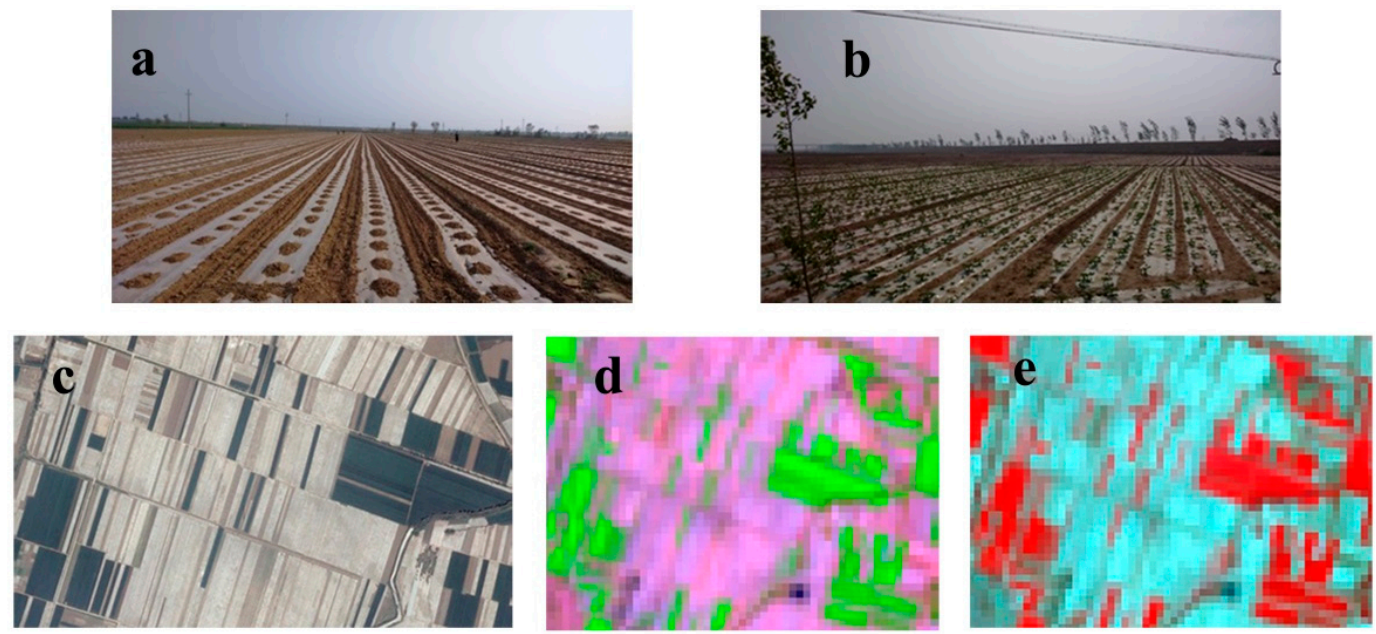

Figure 2. The plastic-mulched farmland in the study area: (a) field photo of plastic-mulched farmland at sowing stage; (b) field photo of plastic-mulched farmland at seedling stage; (c) plastic-mulched farmland in the Google Earth imagery; (d) plastic-mulched farmland presented in false color composite Landsat-8 OLI imagery: $R$ = SWIR2 (Shortwave Infrared 2), G = NIR, B = red; and (e) plastic-mulched farmland presented in false color composite Landsat- 8 OLI imagery: $R=N I R, G=$ red, $B=$ green.

\subsection{Data}

\subsubsection{Remote Sensing Imagery}

Data used in this paper include Landsat-8 OLI imagery, Google Earth imagery, and ancillary data. The Landsat- 8 satellite carries two sensors: the OLI and the TIRS (Thermal Infrared Sensor). The OLI provides imagery data every 16 days in eight medium spatial resolution $(30 \mathrm{~m})$ shortwave (Visible-near-infrared, Shortwave Infrared) bands (Coastal Aerosol, Blue, Green, Red, NIR, SWIR1 (Shortwave Infrared 1), SWIR2 and Cirrus) and one $15 \mathrm{~m}$ panchromatic band. The TIRS offers two types of longwave thermal-infrared data imagery with $100 \mathrm{~m}$ spatial resolution.

The temporal characteristic of remote sensing data is a key factor for monitoring ground objects because the remote sensing signature changes over time and space. The spectral characteristic of plastic-mulched farmland is influenced greatly by mulched crops, dust and rainfall after it has been deployed; thus the optimal monitoring period is just after deployment. However, the deployment time differs across different regions and different crops. The cotton (the only plastic-mulched crop in Jizhou) is planted from mid-to-late April and seedlings emerge between late April and early May. At this time of year, winter wheat is at the jointing and heading stage, while corn is sowed in early June. Thus, the optimal period for monitoring plastic-mulched cotton field is from mid-to-late April to early May. Therefore, we chose to use the OLI imagery acquired on 29 April 2014 as the main remote sensing data. The OLI imagery for path/row 114/27 was downloaded from USGS GLOVIS, which is a high-quality L1T-type product (free of clouds) that has been geometrically corrected. To obtain more accurate input satellite imagery for monitoring plastic-mulched farmland, radiometric calibration and atmospheric correction with Fast Line of-sight Atmospheric Analysis of Spectral Hypercubes (FLAASH) were performed. The Google Earth imagery was acquired on 29 April 2014 also, and was used to collect samples for classification.

\subsubsection{Classification Scheme and Ground Truth Samples}

To meet the research requirement and simultaneously stay consistent with the actual spatial pattern of the study area, a classification scheme that included five main land cover classes were established (Table 1). There are some rare greenhouse distributions that did not meet the required number of training and testing samples, so greenhouses were masked using data from visual 
interpretations of the high spatial resolution Google Earth imagery. The classifiers adopted in this paper were supervised classification methods, which require sufficient numbers of training and testing samples. Stratified random sampling was performed to avoid missing rare types of samples and to ensure that the selected samples were uniform and representative of the study space and types [18]. The samples ( 3 pixels $\times 3$ pixels sized) for each class were collected separately by visually interpreting the Google Earth imagery and the Landsat-8 OLI imagery using stratified random sampling methods. A total of 839 random samples (7551 pixels) were obtained. Then the samples were systemically divided in half for training and testing. The number of training and testing samples was kept roughly equal both infraclass and interclass (except for the rare distribution classes).

Table 1. The land cover classification scheme.

\begin{tabular}{|c|c|c|c|}
\hline Initial Classes & Abbreviation & Remarks & Final Classes \\
\hline $\begin{array}{l}\text { Plastic-Mulched } \\
\text { Farmland }\end{array}$ & PMF & White Plastic Film & PMF \\
\hline Impervious Surface & IS & Buildings, Factories, Roads and Dam Boundaries & \multirow{4}{*}{ Non-PMF } \\
\hline Vegetation Cover & $\mathrm{VC}$ & Crop, Vegetable Field, Grassland, Woodland & \\
\hline Water Body & WB & Rivers, Lakes and Irrigation Canals & \\
\hline Bare Soil & BS & Bare Land, Fallow land and Abandoned Land & \\
\hline
\end{tabular}

\section{Methods}

\subsection{Feature Extraction and Selection Methods}

Feature extraction and selection play an important role in imagery classification or object detection tasks. Based on certain evaluation criteria, an optimal or most effective feature subset is selected from the original feature set to reduce the dimensionality of the feature space. Via feature selection, relevant or redundant features will be removed from the original space; only the important features will be preserved [19].

\subsubsection{Spectral Separability Analysis}

From the spectral separability of the five land cover classes (Figure 3), we can see that plastic-mulched farmland is well separated on the visible and near infrared bands. On the visible bands, these classes are clustered into three groups: plastic-mulched farmland, impervious surface and bare soil, vegetation and water bodies. Although plastic-mulched farmland and bare soil are not well separated on the shortwave infrared bands, the impervious surfaces and bare soil are well separated. The reflectance of plastic-mulched farmland is higher than the other classes on the visible and shortwave infrared bands. The reflectance of vegetation is highest on the near infrared band. Using the seven bands of OLI imagery, all five classes are well separated.

Plastic film is made of polyethylene, a white, semitransparent, nonpolar thermoplastic resin with high crystallinity. The spectral reflectance of plastic-mulched farmland depends largely on the soil characteristics. Thus, the shape of the spectral curve of plastic-mulched farmland is similar to the spectrum curve shape of the soil. Soil reflectance generally increases as the wavelength increases. However, plastic-mulched farmland is brighter, smoother (because it reduces soil roughness) and drier (it has low vapor and air permeability and low water absorbability) than other classes, so the reflectance of plastic-mulched farmland is also higher than those other classes on visible shortwave infrared bands. On the near infrared band, the highest reflectance accrues to repeated reflections between leaf cell walls and the cell gap of green vegetation. The OLI Data used in this paper were acquired at the sowing stage (29 April 2014) for plastic-mulched cotton. Using this date, we can avoid most of the effects of vegetation, dust and rainwater on plastic-mulched farmland that would occur following plastic mulch deployment. The spectrum signature of plastic-mulched farmland is captured well by this remotely sensed data. 


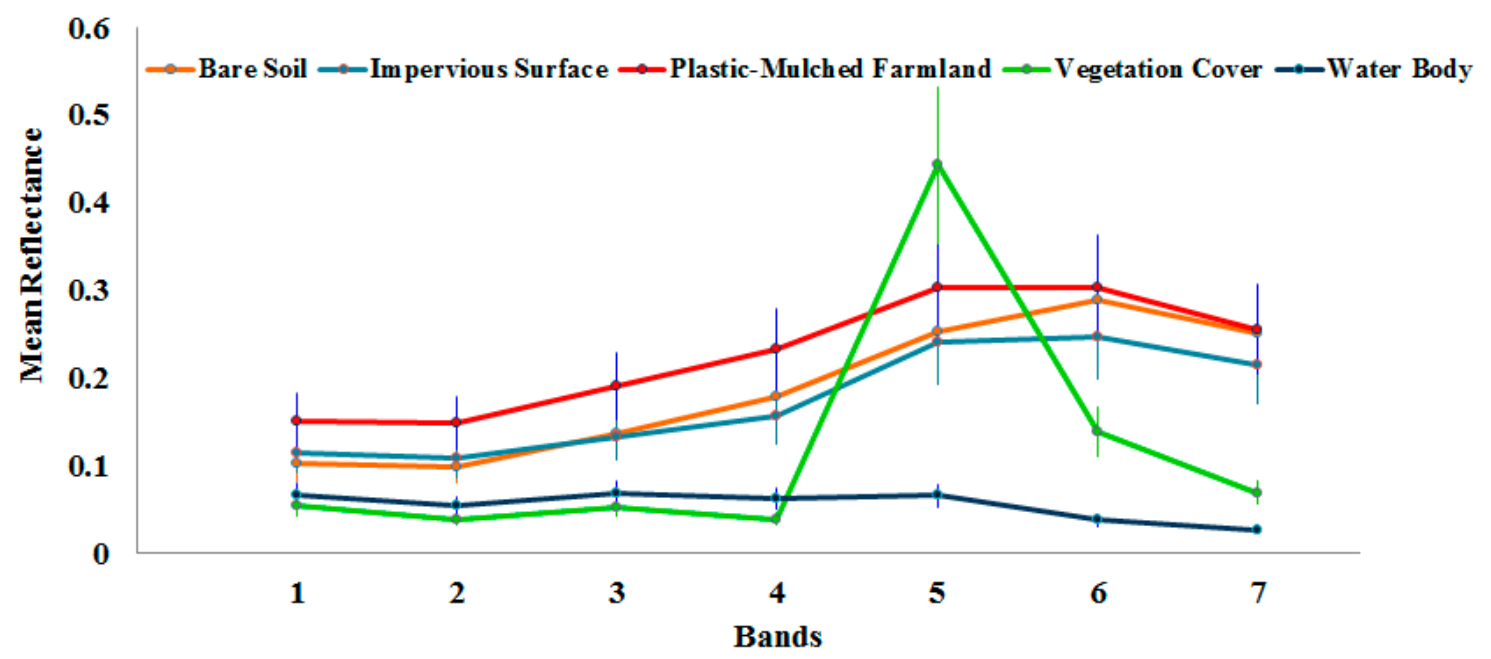

Figure 3. Spectral separability of different land cover classes on the different bands of Landsat-8 OLI imagery (1: Coastal Aerosol; 2: Blue; 3: Green; 4: Red; 5: NIR; 6: SWIR1; 7: SWIR2).

\subsubsection{Textural Features Extraction and Selection}

Texture analysis has been widely used in remote sensing classification [20-23]. The (GLCM) proposed by Haralick in 1973 [24] has become one of the most common and widely accepted statistical texture analysis methods [25]. The GLCM is a function of both the direction and distance relationship between two neighboring pixels. It records the number of co-occurrence relationships between one pixel and its specified neighbor pixels for a specified direction and distance. Several studies have used the GLCM method for classification and obtained desired results [26-34].

In this paper, the GLCM were calculated in four different directions $\left(\theta=0^{\circ}, 45^{\circ}, 90^{\circ}\right.$ and $\left.135^{\circ}\right)$ and over three different distances $(\mathrm{d}=1,2,3$ pixels) using the multi-bands data. Considering the spatial distribution pattern of plastic-mulched farmland and the spatial resolution of the imagery, we did not consider or design different window sizes. One window size ( 3 pixels by 3 pixels) was defined for calculating textures. The widely used textural features of mean, variance, homogeneity, contrast, dissimilarity, entropy, angular second moment and correlation were calculated using the GLCM. Mean describes the levels of shades of gray; variance reflects the uneven characteristics of gray levels; correlation describes similarity at a certain position; and contrast, dissimilarity and homogeneity reflect the comparability of the imagery. Contrast is the total variance of gray level within small area, which becomes greater when the gray level difference is larger. Dissimilarity is a measurement of changing degree of gray level. There are positive correlations between contrast and dissimilarity; and negative correlations between contrast and homogeneity. Angular second moment, also called energy, is a measurement of the distribution uniformity of grayscale. The greater the value of angular second moment is, the coarser the texture is. There are inverse correlations between angular second moment and entropy. When there are more classes distributed at uneven gray levels, the textures are mixed and disordered, and the angular second moment is smaller, the entropy is quite larger. In contrast, when there are fewer classes, the angular second moment is greater, and the entropy is quite small.

Many of the textural features measured using multi-bands may be highly correlated or redundant [35] because the textural features are derived from the spectral information. Moreover, textural features obtained from different directions and distances on seven bands of Landsat-8 OLI imagery imply large increases in feature dimensionality. High dimensionality in the feature set may increase computational time, overwhelm the classifier, and result in "curse of dimensionality" [36]. Thus, the most valuable textural features should be selected prior to performing the classifiers to speed up the training process and increase interpretability by alleviating the "curse of dimensionality". Feature selection is an effective approach for alleviating "curse of dimensionality" and speeding up computational time and for developing a robust and independent feature subset for imagery 
classification [37]. Many approaches have been presented for feature selection such as principal component analysis [38,39]; more recently, non-parametric algorithms such as neural network, support vector machine and classification tree $[40,41]$ have been used widely. These approaches can be broadly divided into three categories: filters, wrappers and embedded methods. The advantage of embedded methods is that feature selection is completed during the learning process. The Random Forests (RF) feature selection method is a decision tree-based ensemble method that is efficient computationally, robust to outliers and noise, and useful for estimating error, strength, correlation, and variable importance [42].

In this paper, the 672 GLCM textural features were obtained from seven bands of Landsat-8 OLI imagery in four directions and three different pixel distances. The importance of textural features was subsequently calculated using the random forest feature selection method in four directions. The top 20 textural features with importance value greater than 1 were selected in the four directions, respectively. The random forest algorithm is characterized by the random selection of samples and features, and the results of random selection are little different across different runs. In order to get more stable and accurate feature sets, the random forest feature selection process has been repeated for many times in our study. The differences between runs were mainly in the order of feature importance, not in feature types, and the top 20 selected textural features remained generally consistent across different runs. Then, the top 20 selected textural features and the spectral features were combined to develop various input feature sets for monitoring plastic-mulched farmland. In total, six different feature sets were generated using the following combinations:

(1) Spectral features (S): Include spectral reflectance of the 7 bands of Landsat-8 OLI imagery;

(2) Spectral features + textural features $1\left(S+T_{1}\right)$ : Include spectral features and textural features in the $0^{\circ}$ direction;

(3) Spectral features + textural features $2\left(S+T_{2}\right)$ : Include spectral features and textural features in the $45^{\circ}$ direction;

(4) Spectral features + textural features $3\left(\mathrm{~S}+\mathrm{T}_{3}\right)$ : Include spectral features and textural features in the $90^{\circ}$ direction;

(5) Spectral features + textural features $4\left(\mathrm{~S}+\mathrm{T}_{4}\right)$ : Include spectral features and textural features in the $135^{\circ}$ direction; and

(6) Spectral features + textural features $5\left(S+T_{5}\right)$ : Include spectral features and textural features in all directions.

\subsection{Classification and Accuracy Assessment Methods}

\subsubsection{Classification Methods}

In this paper, we selected three supervised classification algorithms: a non-parametric Support Vector Machine (SVM) with four different kernel functions, and parametric classifiers: the Maximum Likelihood Classifier (MLC) and Minimum Distance Classifier (MDC), because they are extensively used and perform well in remote sensing. The MLC and the MDC were the benchmarks used to compare the non-parametric and parametric classifiers in this paper. These three classifiers are briefly described below.

The SVM algorithm is well-known in the machine learning field and has been successfully used in a wide range of classification problems in remote sensing [43]. Various studies have shown that the SVM classifier outperforms other classifiers [44-50]. The concept underlying SVM is based on structural risk minimization and separating margin maximization [51]. Several different kinds of kernel functions, such as linear kernel function (SVM-L), radial basis kernel function (SVM-R), polynomial kernel function (SVM-P) and sigmoid kernel function (SVM-S), are used to generalize the nonlinear decision boundaries in SVM. Linear kernel function does not need parameters to transform the data; the polynomial kernel function needs a simple nonlinear parameter to transform the data; the sigmoid kernel generates the support vector machine model using similar to neural network $\mathrm{S}$ 
type activation function; and the radial basic function is known as effective for many types of data. The assumption of the MLC method is that the statistics of each class in each band are distributed normally. The probability that a given pixel belongs to a specific class is calculated. Then, each pixel is classified into a specific class with the highest probability (that is, the maximum likelihood) [52]. The MDC calculates the Euclidean distance from each unknown pixel for each class using the mean vectors of each endmember. All pixels are classified into the nearest class.

\subsubsection{Accuracy Assessment}

The measures of overall accuracy, kappa coefficient, producer's accuracy and user's accuracy were used to assess the performance of the classifiers. The overall accuracy is defined as the ratio of the total correctly classified pixels to the total number of pixels (the total number of all ground truth reference pixels). The producer's accuracy corresponds to omission error, while the user's accuracy corresponds to commission error.

\section{Results and Discussions}

\subsection{Results}

\subsubsection{The Spatial Distribution of Plastic-Mulched Farmland}

Landsat-8 OLI imagery can provide valuable information for monitoring plastic-mulched farmland, due to the spectral, spatial and temporal characteristics of plastic-mulched farmland and the imagery. The results (Figure 4) shown that the plastic-mulched farmland in Jizhou, Hebei Province is distributed primarily among the middle, north and south, and dispersed in the east and west. The spatial distributions of the plastic-mulched farmland obtained from these classifiers match well with the field survey results of the same area. There are marginal differences between the results of SVM using different kernel functions and larger differences between the SVM results and those of MLC or MDC using different input feature sets. In this paper, the fewer results of classifiers (SVM-L and the MLC using different feature sets) were taken as examples to display the differences between classifiers and feature sets (Figure 4). From the figure, we can see that there exist a certain degree of differences between classifiers and between feature sets. The spatial distribution from conventional supervised classifier (MLC) is more extensive than that from machine learning algorithm SVM. The results from spectral features are more scattered than that from the combined spectral and textural features. 


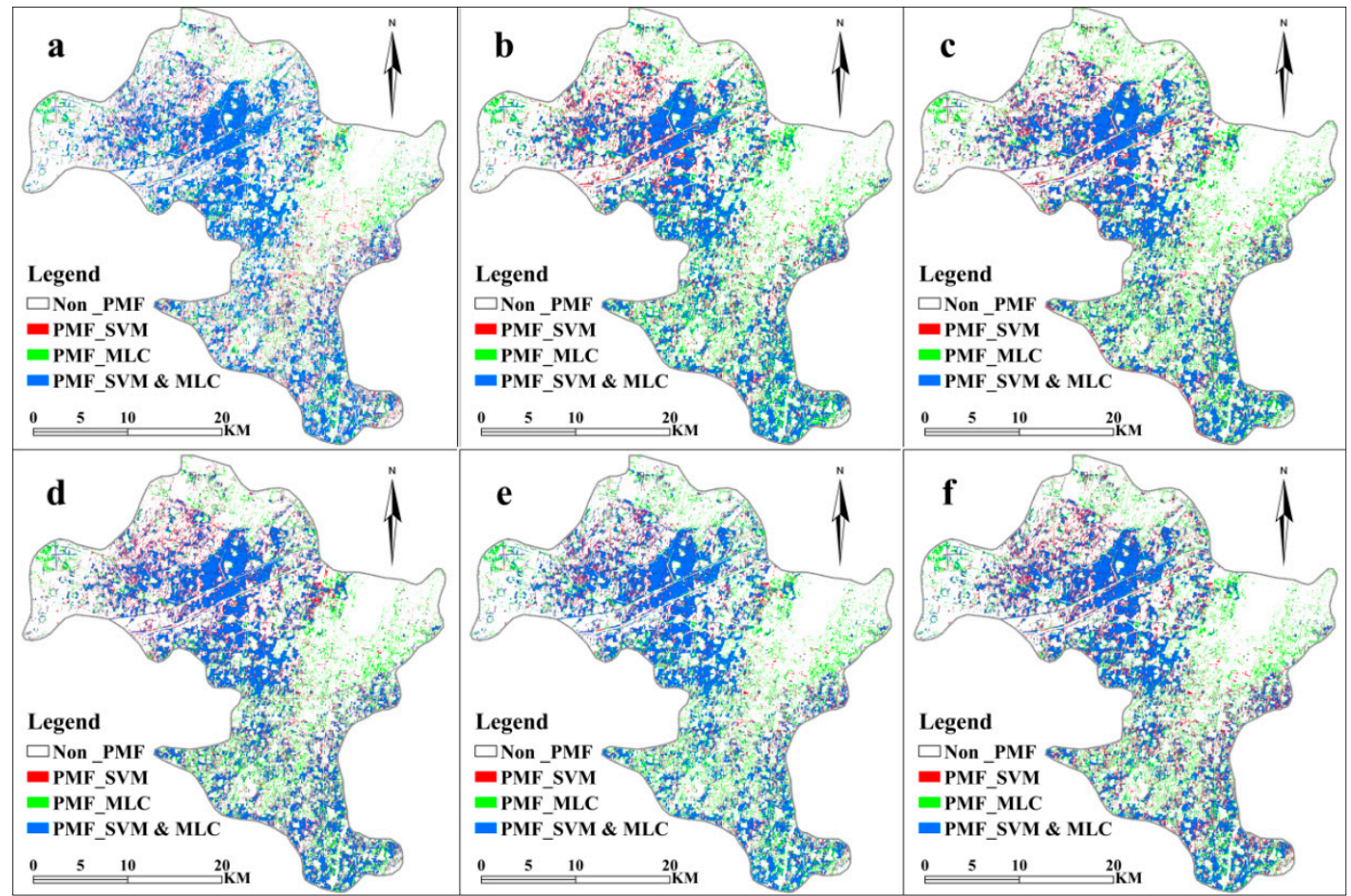

Figure 4. The spatial distribution of the plastic-mulched farmland extracted from different classifiers using different feature sets (Non_PMF: Non plastic-mulched farmland area; PMF_SVM: plastic-mulched farmland extracted from SVM-L alone and not from MLC; PMF_MLC: plastic-mulched farmland extracted from MLC alone and not from SVM-L; PMF_SVM \& MLC: plastic-mulched farmland extracted from both SVM-L and MLC): (a) results of spectral features; (b) results of combined spectral features and $0^{\circ}$ directional textural features; (c) results of combined spectral features and $45^{\circ}$ directional textural features; (d) results of combined spectral features and $90^{\circ}$ directional textural features; (e) results of combined spectral features and $135^{\circ}$ directional textural features; and (f) results of combined spectral features and all directional textural features.)

\subsubsection{Accuracy Assessment}

From the classification accuracies, we found that plastic-mulched farmland can be extracted effectively from Landsat-8 OLI imagery using different classifiers and feature sets. On the whole, the non-parametric machine learning classifier (SVM) outperforms the parametric classifiers (MLC and MDC). In some cases, the overall accuracy of MLC was higher than SVM, but the producer's and user's accuracies for plastic-mulched farmland obtained from SVM were more stable than those from the parametric classifiers MLC and MDC while keeping the accuracy levels equal.

The SVM performs well with all four kernel functions for monitoring plastic-mulched farmland using combined spectral and textural features from Landsat-8 OLI imagery. The accuracies of all the combinations of different SVM kernel functions and input feature sets were beyond 92.7\%, and both the producer's accuracy and user's accuracy for detecting plastic-mulched farmland were higher than $88.82 \%$ (SVM-S using $\mathrm{S}+\mathrm{T}_{1}$ feature). However, there were a few differences between the various kernel functions and input feature sets. Generally, the linear kernel function provided better results, followed by radial basis kernel function and the polynomial kernel function. From the difference value (Figure 5) between the accuracy of combined spectral and textural features $\left(\mathrm{S}+\mathrm{T}_{1} / \mathrm{S}+\mathrm{T}_{2} / \mathrm{S}+\mathrm{T}_{3} / \mathrm{S}+\mathrm{T}_{4} / \mathrm{S}+\mathrm{T}_{5}\right)$ and the accuracy of the spectral feature $(S)$ alone, we can see that the overall, producer's and user's accuracies of SVM-L were increased when including textural features in the $90^{\circ}$ direction. The accuracy of SVM-R was increased also when including textural features calculated in the $45^{\circ}$ direction, while the increment in the other direction were unstable (it both increases and decreases). The highest overall 
accuracy for SVM with the linear kernel function was derived from the combined use of spectral and textural features in the $90^{\circ}$ direction (94.14\%, kappa 0.92), followed by using combined spectral and textural features in the $45^{\circ}(93.84 \%$, kappa 0.92$), 135^{\circ}(93.73 \%$, kappa 0.92$)$ and $0^{\circ}(93.71 \%$, kappa 0.92$)$ directions, and finally, the spectral features alone (93.57\%, kappa 0.91). Improved producer's and user's accuracies for plastic-mulched farmland were obtained from combined spectral features and textural features in the $90^{\circ}$ direction, $90.67 \%$ (90.58\%), followed by combined spectral features and textural features in the $0^{\circ}$ and $135^{\circ}$ direction, $91.06 \%(90.53 \%)$ and $90.67 \%(90.23 \%)$, respectively, the spectral features alone, $90.38 \%(90.38 \%)$, and combined spectral features and textural features in the $45^{\circ}$ direction, $90.28 \%(90.19 \%)$, and finally, combined spectral features and textural features in the all directions, $90.28 \%(89.76 \%)$.

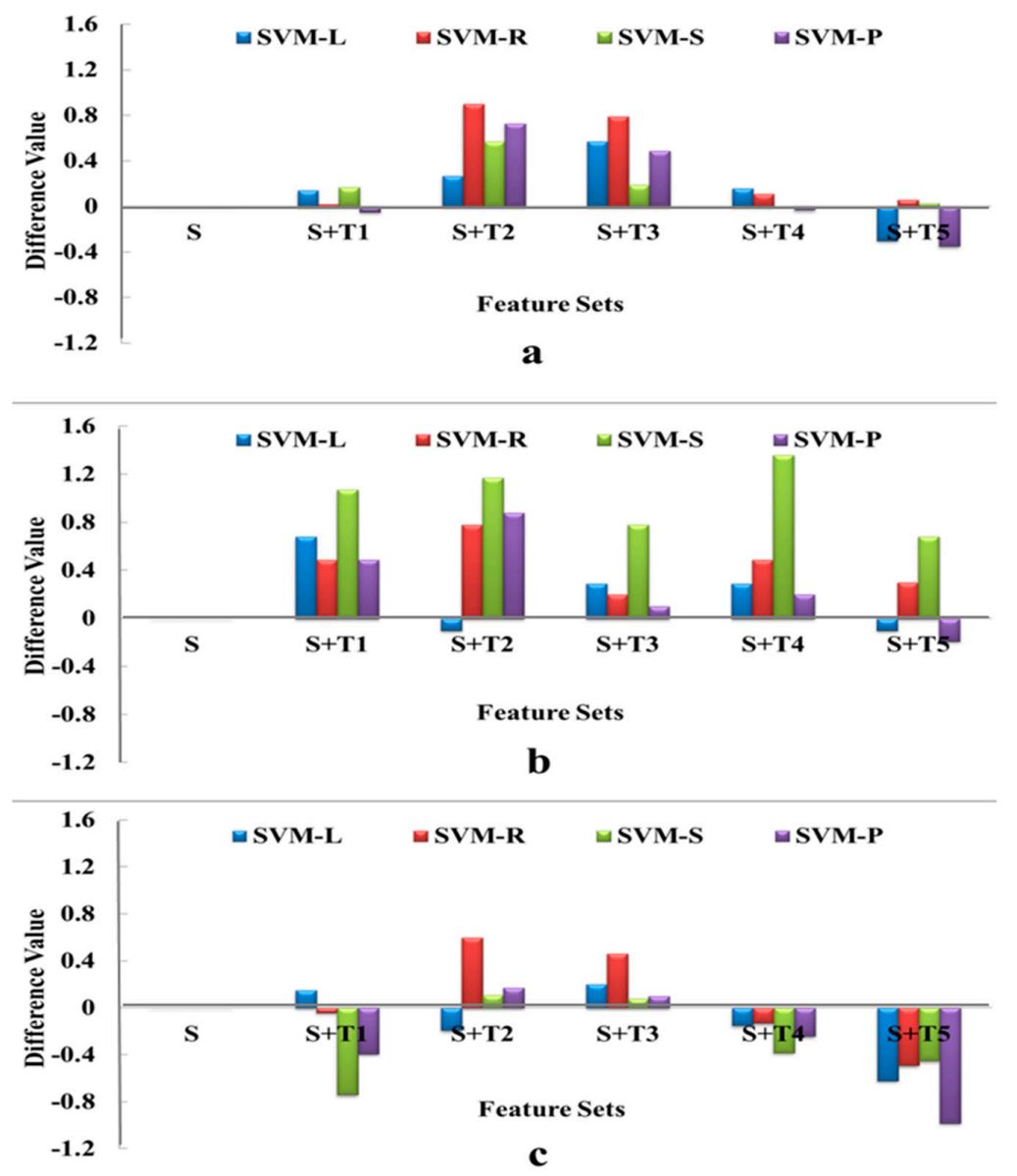

Figure 5. The accuracy changes of different kernel functions SVM using different feature sets: (a) the difference value of overall accuracy; (b) the difference value of producer accuracy; and (c) the difference value of user accuracy; Difference Value $=$ Accuracy $\left._{(S+T 1 / S+T 2 / S}+\mathrm{T} 3 / \mathrm{S}+\mathrm{T} 4 / \mathrm{S}+\mathrm{T} 5\right)-$ Accuracys $)$.

The inclusion of textural features improves accuracy slightly when the SVM kernel functions and the input feature sets are optimized. In our examinations, classification accuracies from different combinations of spectral features and textural features were only marginally different from each other; the combined spectral features and textural features in the $90^{\circ}$ direction using SVM with the linear kernel function was achieved the highest accuracy.

From the confusion matrix (Table 2) among plastic-mulched farmland, impervious surface and bare soil (there was no confusion between water body, vegetation cover and the other classes, so these two land cover classes were not presented in Table 2) of SVM-L and MLC, it can be observed that confusion between plastic-mulched farmland and bare soil was more serious than between plastic-mulched farmland and impervious surfaces. The misclassification percentage from bare soil 
to plastic-mulched farmland was higher than the misclassification percentage from plastic-mulched farmland to bare soil in most cases. The misclassification percentage from impervious surfaces to plastic-mulched farmland was higher than the percentage from plastic-mulched farmland to impervious surfaces in most cases as well. These misclassifications have the potential to increase the area of classified plastic-mulched farmland, which would also influence the confidence level in its spatial distribution. The plastic film used for mulching is very thin $(0.008 \mathrm{~mm})$, and it is deposited closely over the soil surface; therefore, the spectral information acquired by the sensor is a confused electronic signature of both soil and plastic film. The $1 \mathrm{~m}$ width plastic-mulched sections are separated by narrower bare soil rows distributed alternately in plastic-mulched cotton fields, making mixed pixels extremely common in medium resolution imagery.

Table 2. Confusion matrix of parts land cover classes (PMF, IS and BS) from SVM-L and MLC.

\begin{tabular}{cccccccc}
\hline \multirow{2}{*}{ Feature Sets } & Classifiers & \multicolumn{3}{c}{ SVM-L } & \multicolumn{3}{c}{ MLC } \\
\cline { 2 - 8 } & Land Cover Classes & PMF & IS & BS & PMF & IS & BS \\
\hline \multirow{3}{*}{$\mathrm{S}$} & PMF & 90.38 & 1.98 & 12.44 & 88.63 & 1.21 & 13.36 \\
& IS & 0.49 & 95.27 & 2.00 & 0.49 & 96.92 & 1.54 \\
& BS & 9.14 & 2.64 & 85.56 & 10.88 & 1.65 & 85.10 \\
\hline \multirow{3}{*}{$\mathrm{S}+\mathrm{T}_{1}$} & PMF & 91.06 & 1.98 & 12.29 & 88.82 & 1.32 & 14.29 \\
& IS & 0.39 & 95.27 & 2.46 & 0.39 & 97.69 & 1.23 \\
& BS & 8.55 & 2.09 & 85.25 & 10.79 & 0.66 & 84.33 \\
\hline \multirow{3}{*}{$\mathrm{S}+\mathrm{T}_{2}$} & PMF & 90.28 & 1.98 & 12.75 & 85.71 & 1.32 & 10.14 \\
& IS & 0.97 & 96.81 & 1.54 & 0.68 & 96.92 & 1.23 \\
& BS & 8.75 & 1.21 & 85.71 & 13.61 & 1.65 & 88.63 \\
\hline \multirow{3}{*}{$\mathrm{S}+\mathrm{T}_{3}$} & PMF & 90.67 & 1.43 & 12.9 & 88.24 & 1.32 & 12.29 \\
& IS & 1.17 & 97.25 & 1.08 & 0.58 & 95.93 & 1.54 \\
& BS & 8.16 & 1.32 & 86.02 & 11.18 & 2.64 & 86.18 \\
\hline \multirow{3}{*}{$\mathrm{S}+\mathrm{T}_{4}$} & PMF & 90.67 & 1.87 & 12.9 & 88.63 & 0.88 & 12.75 \\
& IS & 0.29 & 95.71 & 1.84 & 0.10 & 97.14 & 2.00 \\
& BS & 9.04 & 2.31 & 85.25 & 11.27 & 1.87 & 85.25 \\
\hline \multirow{2}{*}{$\mathrm{S}+\mathrm{T}_{5}$} & PMF & 90.28 & 1.98 & 13.52 & 84.84 & 0.66 & 11.21 \\
& IS & 0.58 & 95.60 & 2.92 & 0.39 & 97.14 & 1.23 \\
& BS & 9.14 & 2.20 & 83.56 & 14.77 & 1.98 & 87.56 \\
\hline
\end{tabular}

\subsection{Discussion}

From these results, we can conclude that the highest accuracy in detecting plastic-mulched farmland was obtained from the SVM using linear kernel function and combined spectral and textural features in the $90^{\circ}$ direction. Thus, the following discussion focuses on this combination of feature sets and method.

\subsubsection{The Contribution of Textural Features}

To explain the effects of spectral and spatial characteristics of textural features for monitoring plastic-mulched farmland, we conducted comparative analyses using textural features alone from various bands and a combination of spectral and textural features using SVM-L. Textural features include those calculated from the panchromatic band $\left(\mathrm{T}_{\mathrm{P}}\right)$, pan-sharpened multi-bands $\left(\mathrm{T}_{\mathrm{F}}\right)$, and original bands $\left(\mathrm{T}_{3}\right)$ in the $90^{\circ}$ direction. We also compare the accuracy among the seven feature sets: the spectral features alone $(S)$, original multi-bands textural features alone $\left(\mathrm{T}_{3}\right)$, pan-sharpened multi-bands textural features alone $\left(\mathrm{T}_{\mathrm{F}}\right)$, panchromatic band textural features alone $\left(\mathrm{T}_{\mathrm{P}}\right)$, and combined spectral and original multi-bands textural features $\left(S+T_{3}\right)$, combined spectral and pan-sharpened multi-bands textural features $\left(S+T_{F}\right)$, and combined spectral and panchromatic band textural features $\left(S+T_{P}\right)$. 
From the results (Table 3), we can see that both the spectral features and the multi-bands textural features (except for the panchromatic band textural features) can serve to detect plastic-mulched farmland. However, using spectral features alone provided higher accuracy than using textural features alone. Among the textural features, the panchromatic band textural features alone provided lower overall accuracy than the other two textural features, and there were similar accuracy levels between the original multi-bands textural features alone $\left(\mathrm{T}_{3}\right)$ and the pan-sharpened multi-bands textural features alone $\left(\mathrm{T}_{\mathrm{F}}\right)$.

The accuracy derived from the combined spectral and textural feature sets was higher than that from spectral or textural features alone. Among the combined feature sets, the overall accuracy achieved by $S+T_{3}$ was higher than the other two combined feature sets. In addition, the producer's and user's accuracies achieved by $S+T_{F}$ were higher than accuracies for the other feature sets. However, the improvement in accuracy was both limited and almost negligible. Because the textural features are second-level features derived from the spectral reflectance, there may be information redundancy in the combined spectral and textural features; therefore, accuracy is not improved significantly by the addition of textural features.

Table 3. The classification accuracy of different features sets.

\begin{tabular}{|c|c|c|c|c|}
\hline Feature Sets & Abbreviations & Overall Accuracy & Producer's Accuracy & User's Accuracy \\
\hline Spectral Features & $\mathrm{S}$ & 93.57 & 90.38 & 90.38 \\
\hline Texture from Original Multi-Bands & $\mathrm{T}_{3}$ & 92.89 & 89.31 & 90.10 \\
\hline Texture from Pan-Sharpened Multi-Bands & $\mathrm{T}_{\mathrm{F}}$ & 92.93 & 91.07 & 90.22 \\
\hline $\begin{array}{l}\text { Combined Spectral and Original } \\
\text { Multi-Bands Textural Features }\end{array}$ & $\mathrm{S}+\mathrm{T}_{3}$ & 94.14 & 90.67 & 90.58 \\
\hline $\begin{array}{c}\text { Combined Spectral and Pan-Sharpened } \\
\text { Multi-Bands Textural Features }\end{array}$ & $\mathrm{S}+\mathrm{T}_{\mathrm{F}}$ & 93.68 & 91.63 & 91.15 \\
\hline
\end{tabular}

\subsubsection{Stability of SVM}

The stability of a classifier is always influenced by the samples and feature sets. In this section, we discussed the effects of the number and distribution of samples and feature sets for SVM using the linear kernel function.

\section{The Number and Distribution of Samples}

The number and distribution of the training and testing samples are important for classification accuracy. For this study, five-fold cross validations were conducted. All the samples of each class were divided into five equal sets, with training being conducted on four sets and testing on one set; this was repeated five times and the mean accuracy was taken. The mean accuracy of $94.41 \%$ was achieved using SVM with the linear kernel function. From the results, we can conclude that, on the one hand, the distribution and number of the training and testing samples influence the classification accuracy to some degree but not significantly; on the other hand, the SVM with the linear kernel function is a relatively stable classifier for our study area.

\section{The Number of Features}

We developed three different combined feature sets (spectral features and top 10 textural features, spectral features and top 15 textural features, and spectral features and top 20 textural features, all calculated in the $90^{\circ}$ direction) to discuss the effects of the number of feature sets required for classifier stability. From the accuracy (Table 4), we can see that the overall, producer's and user's accuracies improved slightly as the input feature sets were increased from combined spectral and top 10 textural features to top 15 textural features, and to top 20 textural features. However, this feature-dimensionality 
increment has almost no significant effect on the performance of SVM-L. SVM-L is both effective and insensitive to feature dimensionality. The SVM outperformed the conventional classifier as well.

Table 4. The accuracy of SVM-L using different number of features.

\begin{tabular}{|c|c|c|c|c|c|}
\hline Classifier & $\begin{array}{l}\text { Feature } \\
\text { Sets }\end{array}$ & $\begin{array}{c}\text { Overall } \\
\text { Accuracy }\end{array}$ & $\begin{array}{c}\text { Producer's } \\
\text { Accuracy }\end{array}$ & $\begin{array}{c}\text { User's } \\
\text { Accuracy }\end{array}$ & $\begin{array}{c}\text { Kappa } \\
\text { Coefficient }\end{array}$ \\
\hline \multirow{3}{*}{ SVM-L } & $\mathrm{S}+\mathrm{Top}_{10}$ & 93.71 & 90.67 & 90.14 & 0.91 \\
\hline & $\mathrm{S}+\mathrm{Top}_{15}$ & 93.90 & 90.96 & 90.43 & 0.92 \\
\hline & $\mathrm{S}+\mathrm{Top}_{20}$ & 94.14 & 90.67 & 90.58 & 0.92 \\
\hline
\end{tabular}

\section{Conclusions}

In this paper, Landsat-8 OLI multispectral data were evaluated to determine their suitability for monitoring plastic-mulched farmland in Jizhou, Hebei Province, China. In addition, the performance of the non-parametric classifier SVM with different kernel functions and the parametric classifiers MLC and MDC were tested. The contribution of textural features was also discussed in monitoring plastic-mulched farmland. We can conclude that:

(1) Plastic-mulched farmland in Jizhou can be effectively extracted from medium spatial resolution Landsat-8 OLI data. The methods used here achieved high overall, producer's and user's accuracies (above $85 \%$ ) using various methods and different feature sets.

(2) SVM performed well with linear, radial basis kernel function, polynomial and sigmoid kernel functions, although SVM with linear kernel functions provided the best performance. Overall accuracy for the different SVM kernel functions were all beyond 92.7\%, and both the producer's accuracy and user's accuracy scores were higher than $88.82 \%$ (SVM-S using the $\mathrm{S}+\mathrm{T}_{1}$ feature).

(3) The non-parametric classifier SVM with the linear kernel function outperforms the parametric classifiers MLC and MDC for monitoring plastic-mulched farmland. SVM proved to be an effective and relatively stable classifier that was insensitive to feature dimensionality and to the distribution of training and testing samples in our study area.

(4) Both spectral and textural features can describe plastic-mulched farmland sufficiently for detection; however, the spectral features alone provide higher overall accuracy than the textural features alone. Among the textural features, the multi-bands textural features provided higher accuracy than the panchromatic band textural features.

(5) The inclusion of textural features can improve the accuracy of plastic-mulched farmland slightly using the optimized input feature sets. The highest overall accuracy was derived from the combined spectral features and textural features in the $90^{\circ}$ direction $(94.14 \%)$, followed by the combined spectral and textural features in the $45^{\circ}(93.84 \%), 135^{\circ}(93.73 \%)$ and $0^{\circ}(93.71 \%)$ directions and finally, the spectral features alone (93.57\%).

Our study indicated that the SVM classifier is an effective method for monitoring plastic-mulched farmland using Landsat- 8 OLI data. These method and imagery data can be used to monitor and map the spatial distribution and temporal variation of plastic-mulched farmland, can provide basic data for further study of social and eco-environmental effects of plastic-mulched farmland.

Further works should be done to test the multi-temporal Landsat-8 imagery with machine learning algorithms to improve the accuracy of plastic-mulched farmland classification. Furthermore, we plan to explore the optimal spectral and spatial requirements for accurate monitoring plastic-mulched farmland using hyper-spectral and higher spatial resolution remote sensing data.

Acknowledgments: The anonymous reviewers' comments and suggestions greatly improved our paper. We are grateful for their kind help. This work was supported by the National Natural Science Foundation of China (grant No. 41371396) and China Ministry of Agriculture "Introduction of International Advanced Agricultural Science and Technology Program (948 Program)" project (No. 2016-X38). 
Author Contributions: Hasituya and Zhongxin Chen designed and conducted the experiments and contributed extensively in manuscript writing and revision. Limin Wang, Wenbin Wu and Zhiwei Jiang provided suggestions for the experiment design and the data analysis. He Li made efforts in editing the manuscript.

Conflicts of Interest: The authors declare no conflict of interest.

\section{References}

1. Bai, L.; Hai, J.; Han, Q.; Jia, Z. Effects of mulching with different kinds of plastic film on growth and water use efficiency of winter wheat in Weibei Highland. Agric. Res. Arid Areas 2010, 28, 135-139.

2. Takakura, T. Climate under Cover: Digital Dynamic Simulation in Plant Bio-Engineering; Springer Science+Business Media: Berlin, Germany, 1993.

3. Yan, C.; Liu, E.; Shu, F.; Liu, Q.; Liu, S.; He, W. Review of agricultural plastic mulching and its residual pollution and prevention measures in China. J. Agric. Res. Environ. 2014, 31, 95-102.

4. Yan, C.; He, W.; Liu, E.; Lin, T.; Pasquale, M.; Liu, S.; Liu, Q. Concept and estimation of crop safety period of plastic film mulching. Trans. Chin. Soc. Agric. Eng. 2015, 31, 1-4.

5. Yan, C.; Mei, X.; He, W.; Zheng, S. Present situation of residue pollution of mulching plastic film and controlling measures. Trans. Chin. Soc. Agric. Eng. 2006, 22, 269-272.

6. Moreau, S.; Bosseno, R.; Gu, X.F.; Baret, F. Assessing the biomass dynamics of Andean bofedal and totora high-protein wetland grasses from NOAA/AVHRR. Remote Sens. Environ. 2003, 85, 516-529. [CrossRef]

7. Nordberg, M.L.; Evertson, J. Monitoring change in mountainous dry-heath vegetation at a regional scale using multitemporal Landsat TM data. AMBIO 2003, 32, 502-509. [CrossRef] [PubMed]

8. Gao, J. Quantification of grassland properties: How it can benefit from geoinformatic technologies? Int. J. Remote Sens. 2006, 27, 1351-1365. [CrossRef]

9. Haihui, W. Study on the Polarized Reflectance Characteristics of Agricultural Thin Membrane. Master Thesis, Northeast Normal University, Changchun, China, 2007.

10. Lu, L.; Di, L.; Ye, Y. A Decision-Tree classifier for extracting transparent Plastic-Mulched landcover from landsat-5 TM images. IEEE JSTARS 2014, 7, 4548-4558. [CrossRef]

11. Lu, L.; Hang, D.; Di, L. Threshold model for detecting transparent plastic-mulched landcover using moderate-resolution imaging spectroradiometer time series data: A case study in southern Xinjiang, China. J. Appl. Remote Sens. 2015. [CrossRef]

12. Carvajal, F.; Crisanto, E.; Aguilar, F.J.; Aguera, F.A.; Aguilar, M.A. Greenhouses detection using an artificial neural network with a very high resolution satellite image. Proc. ISPRS Tech. Comm. II Symp. 2006. [CrossRef]

13. Aguera, F.; Aguilar, F.J.; Aguilar, M.A. Using texture analysis to improve per-pixel classification of very high resolution images for mapping plastic greenhouses. ISPRS J. Photogramm. Remote Sens. 2008, 63, 635-646. [CrossRef]

14. Aguera, F.; Liu, J.G. Automatic greenhouse delineation from QuickBird and Ikonos satellite images. Comput. Electron. Agric. 2009, 66, 191-200. [CrossRef]

15. Koc-San, D. Evaluation of different classification techniques for the detection of glass and plastic greenhouses from WorldView-2 satellite imagery. J. Appl. Remote Sens. 2013. [CrossRef]

16. Zhao, G.X.; Li, J.; Li, T.; Yue, Y.D.; Warner, T. Utilizing Landsat TM imagery to map greenhouses in Qingzhou, Shandong Province, China. Pedosphere 2004, 14, 363-369.

17. Bin, S. Quality Inspection and Quality Analysis of Hebei Province Jizhou City Environment. Master Thesis, Northwest Agriculture \& Forestry University, Yangling, China, 2014.

18. Zhao, Y. The Principle and Method of Remote Sensing Application; Science Press: Beijing, China, 2003; pp. $204-205$.

19. Fodor, I.K. A survey of dimension reduction techniques. Opt Commun. 2002, 205, 351-359.

20. Li, N.; Bruzzone, L.; Chen, Z.; Liu, F. A novel technique based on the combination of labeled Co-Occurrence matrix and variogram for the detection of built-up areas in High-Resolution SAR images. Remote Sens. 2014, 6, 3857-3878. [CrossRef]

21. Guindon, B.; Zhang, Y.; Dillabaugh, C. Landsat urban mapping based on a combined spectral-spatial methodology. Remote Sens. Environ. 2004, 92, 218-232. [CrossRef]

22. Pesaresi, M.; Gerhardinger, A.; Kayitakire, F. A robust Built-Up area presence index by anisotropic Rotation-Invariant textural measure. IEEE JSTARS 2008, 1, 180-192. [CrossRef] 
23. Pesaresi, M.; Gerhardinger, A. Improved textural Built-Up presence index for automatic recognition of human settlements in arid regions with scattered vegetation. IEEE JSTARS 2011, 4, 16-26. [CrossRef]

24. Ghosh, A.; Joshi, P.K. A comparison of selected classification algorithms for mapping bamboo patches in lower Gangetic plains using very high resolution WorldView 2 imagery. Int. J. Appl. Earth Obs. 2014, 26, 298-311. [CrossRef]

25. Lu, D.; Hetrick, S.; Moran, E. Land cover classification in a complex Urban-Rural landscape with QuickBird imagery. Photogramm. Eng. Remote Sens. 2010, 76, 1159-1168. [CrossRef]

26. Angel Castillo-Santiago, M.; Ricker, M. Estimation of tropical forest structure from SPOT-5 satellite images. Int. J. Remote Sens. 2010, 31, 2767-2782. [CrossRef]

27. Kayitakire, F.; Hamel, C.; Defourny, P. Retrieving forest structure variables based on image texture analysis and IKONOS-2 imagery. Remote Sens. Environ. 2006, 102, 390-401. [CrossRef]

28. Ozdemir, I.; Norton, D.A.; Ozkan, U.Y.; Mert, A.; Senturk, O. Estimation of tree size diversity using object oriented texture analysis and aster imagery. Sensors 2008, 8, 4709-4724. [CrossRef]

29. Pasher, J.; King, D.J. Multivariate forest structure modelling and mapping using high resolution airborne imagery and topographic information. Remote Sens. Environ. 2010, 114, 1718-1732. [CrossRef]

30. Estes, L.D.; Okin, G.S.; Mwangi, A.G.; Shugart, H.H. Habitat selection by a rare forest antelope: A multi-scale approach combining field data and imagery from three sensors. Remote Sens. Environ. 2008, 112, 2033-2050. [CrossRef]

31. Estes, L.D.; Reillo, P.R.; Mwangi, A.G.; Okin, G.S.; Shugart, H.H. Remote sensing of structural complexity indices for habitat and species distribution modeling. Remote Sens. Environ. 2010, 114, 792-804. [CrossRef]

32. Beekhuizen, J.; Clarke, K.C. Toward accountable land use mapping: Using geocomputation to improve classification accuracy and reveal uncertainty. Int. J. Appl. Earth Obs. Geoinf. 2010, 12, 127-137. [CrossRef]

33. Johansen, K.; Coops, N.C.; Gergel, S.E.; Stange, Y. Application of high spatial resolution satellite imagery for riparian and forest ecosystem classification. Remote Sens. Environ. 2007, 110, 29-44. [CrossRef]

34. Pathak, V.; Dikshit, O. A new approach for finding an appropriate combination of texture parameters for classification. Geocarto Int. 2010, 25, 295-313. [CrossRef]

35. Pacifici, F.; Chini, M.E. A neural network approach using multiscale textural metrics from very high-resolution panchromatic imagery for urban land-use classification. Remote Sens. Environ. 2009, 113, 1276-1292. [CrossRef]

36. Bellman, R. Dynamic Programming, 2nd ed.; Dover Publications: Mineola, NY, USA, 2003.

37. Saeys, Y.; Inza, I.; Larranaga, P. A review of feature selection techniques in bioinformatics. Bioinformatics 2007, 23, 2507-2517. [CrossRef] [PubMed]

38. Berberoglu, S.; Curran, P.J.; Lloyd, C.D.; Atkinson, P.M. Texture classification of Mediterranean land cover. Int. J. Appl. Earth Obs. Geoinf. 2007, 9, 322-334. [CrossRef]

39. Bazi, Y.; Melgani, F. Toward an optimal SVM classification system for hyperspectral remote sensing images. IEEE Trans. Geosci. Remote Sens. 2006, 44, 3374-3385. [CrossRef]

40. Pal, M.; Foody, G.M. Feature selection for classification of hyperspectral data by SVM. IEEE Trans. Geosci. Remote Sens. 2010, 48, 2297-2307. [CrossRef]

41. Rodriguez-Galiano, V.F.; Chica-Olmo, M.; Abarca-Hernandez, F.; Atkinson, P.M.; Jeganathan, C. Random Forest classification of Mediterranean land cover using multi-seasonal imagery and multi-seasonal texture. Remote Sens. Environ. 2012, 121, 93-107. [CrossRef]

42. Chen, Y.; Zhao, X.; Lin, Z. Optimizing subspace SVM ensemble for hyperspectral imagery classification. IEEE JSTARS 2014, 7, 1295-1305. [CrossRef]

43. Mountrakis, G.; Im, J.; Ogole, C. Support vector machines in remote sensing: A review. ISPRS J. Photogramm. Remote Sens. 2011, 66, 247-259. [CrossRef]

44. Pal, M.; Mather, P.M. Support vector machines for classification in remote sensing. Int. J. Remote Sens. 2005, 26, 1007-1011. [CrossRef]

45. Melgani, F.; Bruzzone, L. Classification of hyperspectral remote sensing images with support vector machines. IEEE Trans Geosci. Remote Sens. 2004, 42, 1778-1790. [CrossRef]

46. Huang, C.; Davis, L.S.; Townshend, J. An assessment of support vector machines for land cover classification. Int. J. Remote Sens. 2002, 23, 725-749. [CrossRef]

47. Heinzel, J.; Koch, B. Investigating multiple data sources for tree species classification in temperate forest and use for single tree delineation. Int. J. Appl. Earth Obs. Geoinf. 2012, 18, 101-110. [CrossRef] 
48. Angelo, J.J.; Duncan, B.W.; Weishampel, J.F. Using Lidar-Derived vegetation profiles to predict time since fire in an oak scrub landscape in East-Central florida. Remote Sens. 2010, 2, 514-525. [CrossRef]

49. Chen, C.H.; Ho, P.P. Statistical pattern recognition in remote sensing. Pattern Recognit. 2008, 41, $2731-2741$. [CrossRef]

50. Duro, D.C.; Franklin, S.E.; Dube, M.G. A comparison of pixel-based and object-based image analysis with selected machine learning algorithms for the classification of agricultural landscapes using SPOT-5 HRG imagery. Remote Sens. Environ. 2012, 118, 259-272. [CrossRef]

51. Vapnik, V.N. The Nature of Statistical Learning Theory; Springer: New York, NY, USA, 2000.

52. Richards, J.A. Remote Sensing Digital Image Analysis; Springer-Verlag: Berlin, Germany, 1999.

(C) 2016 by the authors; licensee MDPI, Basel, Switzerland. This article is an open access article distributed under the terms and conditions of the Creative Commons Attribution (CC-BY) license (http://creativecommons.org/licenses/by/4.0/). 